\title{
Molecular cloning and expression pattern of the DjStag gene in the planarian Dugesia japonica during embryonic development
}

\author{
Z.Q. Yuan, J.Y. Zhang and B.S. Zhao \\ Laboratory of Developmental and Evolutionary Biology, \\ School of Life Sciences, Shandong University of Technology, Zibo, China \\ Corresponding author: B.S. Zhao \\ E-mail: zhaobosheng@sdut.edu.cn
}

Genet. Mol. Res. 13 (1): 188-197 (2014)

Received May 14, 2013

Accepted November 30, 2013

Published January 10, 2014

DOI http://dx.doi.org/10.4238/2014.January.10.10

\begin{abstract}
We examined STAG-related gene (DjStag) expression in the planarian Dugesia japonica. This species is common in Far Eastern countries. The DjStag cDNA includes 1362 bp and contains a 489-bp open reading frame corresponding to a deduced protein of 162 amino acids, with a 170-bp 5'-UTR and a 703-bp 3'-UTR. Phylogenetic analysis showed that DjStag is an STAG/STAG-like member. We examined the expression pattern of DjStag in this planarian during embryonic development by whole-mount in situ hybridization. DjStag was detected in embryonic cells in the germ band at early embryo stages. The number of DjStag-positive embryonic cells increased in stage 5. Later, it was mainly expressed in lateral region parenchyma. In juveniles, extensive expression of DjStag was observed not only in the head and tail regions, but also in the parenchyma between the epidermis and the gastrodermis. We conclude that DjStag is expressed in the cellular subset that will become the neoblast cells of the adult flatworm. DjStag may play an essential role in spatial and temporal regulation during planarian embryonic development.
\end{abstract}

Key words: Planarian; DjStag; Gene expression; Embryogenesis 


\section{INTRODUCTION}

In eukaryotes, sister chromatids remain physically connected by cohesion from the time of their synthesis in S phase until they are separated in anaphase (Nasmyth et al., 2000; Sumara et al., 2000). Sister chromatid cohesion is a fundamental aspect of chromosome behavior that ensures the accurate inheritance of the genetic information in mitosis and meiosis (Lee and Orr-Weaver, 2001; Nasmyth, 2001; Hirano, 2002). Sister chromatid cohesion depends on cohesin, a conserved protein complex composed of a heterodimeric pair of structural maintenance of chromosome subunits, Smc1 and Smc3, and two sister chromatid cohesion proteins Scc1 and Scc3 (Michaelis et al., 1997; Losada et al., 1998; Sumara et al., 2000). In humans, hoptoad and other higher eukaryotes, two cohesin isoforms exist, each containing the subunits Smc1, Smc3, Scc1, and either one of the Scc3 orthologs STAG1/SA1 or STAG2/SA2 (Losada et al., 2000; Sumara et al., 2000; Prieto et al., 2001).

Previous study on cohesins has been mainly focused on chromatid cohesion and segregation; certain cohesins have been implicated in different cellular processes (Lara-Pezzi et al., 2004). Cohesin is essential not only for chromosome segregation but also double-strand break repair (Ström et al., 2004, 2007; Rubio et al., 2008). Cohesin is also implicated in generegulatory functions (Rubio et al., 2008). The possible function of STAG is not completely understood. Lara-Pezzi et al. (2004) provided evidence suggesting that STAG functions as a transcriptional co-activator by a mechanism involving protein-protein interactions with transcription factors.

Planarians have the ability to regenerate their whole body from any small amputated fragment of their body. Thus, they are excellent models for studying embryonic development and the biology of regeneration (Reddien and Sánchez Alvarado, 2004; Agata et al., 2007; Solana and Romero, 2009). In this study, we isolated and characterized the cDNA sequences of an STAG-related gene (DjStag) from the planarian Dugesia japonica and examined its expression pattern during embryonic development by whole-mount in situ hybridization.

\section{MATERIAL AND METHODS}

\section{Animals and egg capsules}

The planarians and egg capsules used belong to the race of D. japonica collected from a fountain in Quanhetou, Boshan, China. The animals were kept in autoclaved tap water at $20^{\circ} \mathrm{C}$. The embryos were fixed and dissected as previously described (Solana and Romero, 2009). After proper dehydration, embryos could be stored in Eppendorf tubes containing $70 \%$ ethanol at $-20^{\circ} \mathrm{C}$ until used. Juvenile planarians were collected every day and treated with $2 \%$ $\mathrm{HCl}$ for $5 \mathrm{~min}$ on ice, then fixed on ice for $3 \mathrm{~h}$ in Carnoy's fixative (ethanol:chloroform:acetic acid, 6:3:1) and stored in methanol (Palakodeti et al., 2008).

\section{Cloning and sequence analysis of cDNA}

The cDNA library of adult planarians was constructed with the Creator ${ }^{\mathrm{TM}}$ SMART $^{\mathrm{TM}}$ cDNA Library Construction kit (Clontech) according to the method previously described (Liu et al., 2002). In a large-scale sequencing of the cDNA library, more than 1000 clones were 
analyzed for coding probability with the DNATools program. The DjStag cDNA was derived from the planarian cDNA library. Comparison against the GenBank protein database was performed using the BLAST network server at the National Center for Biotechnology Information (NCBI). Phylogenic tree analysis was carried out using the PHYLIP 3.5c software package.

\section{Northern blots}

Northern blotting was carried out as previously described (Palakodeti et al., 2006; Wang et al., 2008). Total RNAs were extracted using the RNAiso Reagent (TaKaRa) according to the manufacturer protocol. The blots were hybridized with DIG-labeled antisense RNA probes at $48^{\circ} \mathrm{C}$ for $16 \mathrm{~h}$. The signal was developed using nitroblue tetrazolium chloride (NBT) and 5-bromo-4-chloro-3'-indolyl phosphate p-toluidine salt (BCIP).

\section{Whole-mount in situ hybridization}

Whole-mount in situ hybridization was performed essentially as previously described (Reddien et al., 2007; Palakodeti et al., 2008). Hybridization was performed at $50^{\circ} \mathrm{C}$ for 36 $\mathrm{h}$ with DIG-conjugated riboprobes in hybridization solution. The color was developed using NBT and BCIP, and the reaction was stopped by two rinses in PBS for 5 min. After bleaching in $5 \% \mathrm{H}_{2} \mathrm{O}_{2}$ in methanol at room temperature under light, whole-mount in situ hybridizations of juveniles were carried out as in dissected embryos. For histological sectioning, some preparations of embryos and juveniles were embedded in paraffin wax and sectioned at $8 \mu \mathrm{m}$ thickness. Whole-mount animals were observed with a Nikon SMZ1500 stereomicroscope and sections were observed with a Zeiss Axioskop-40 microscope.

\section{RESULTS AND DISCUSSION}

\section{Sequence analysis of DjStag}

The cDNA clone (GenBank accession No. GQ503880) obtained from the cDNA library of D. japonica is $1362 \mathrm{bp}$ long, and its longest open reading frame, consisting of 489 $\mathrm{bp}$, codes for a deduced protein of 162 amino acids with a predicted molecular mass of approximately $18.08 \mathrm{kDa}$. Its 5 '-untranslated region (UTR) is $170 \mathrm{bp}$ in length and the 3 '-UTR is 703 bp long with a polyadenylation signal AATAAA and a poly (A) tail (Figure 1). The start codon (ATG) is flanked by a purine base at positions -3 and +4 , matching the Kozak consensus sequence (Kozak, 1987), and is suggested to optimize translational efficiency. Therefore, the cDNA encodes a full-length protein.

Initial BLASTP search at NCBI revealed that the protein encoded by the planarian cDNA shared $49(80 / 163), 39(59 / 151), 37(60 / 158)$, and $37 \%(60 / 158)$, identity to STAG/STAG-like protein of the schistosome, Schistosoma mansoni (GenBank accession No. CAZ30922); drosophila, Drosophila melanogaster (CAA74654); cow, Bos taurus (XP_001249706); human, Homo sapiens (NP_001036214), respectively. This similarity of the primary amino acid sequence suggested that the gene was a planarian STAG-like gene. Therefore, the cDNA appeared to code for a planarian STAG-like protein and was designated DjStag in this paper. 


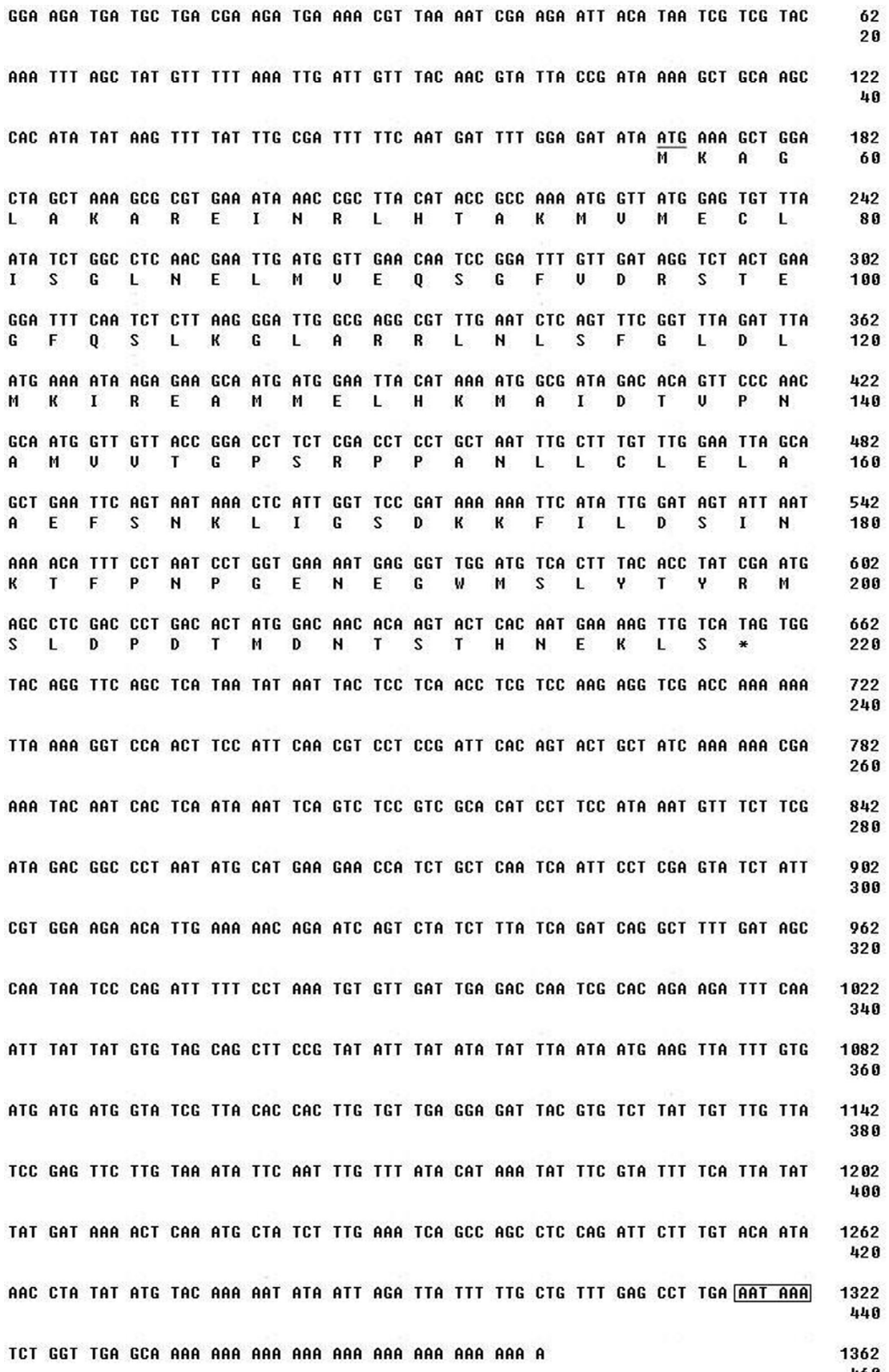

Figure 1. Nucleotide and deduced amino acid sequences of planarian (Dugesia japonica) DjStag cDNA (GenBank accession No. GQ503880). The start codon is underlined. The asterisk represents the stop codon. The polyadenylation signal is boxed. The numbering of the nucleotide and amino acid sequences is shown to the right. 
To shed light on the evolutionary position of DjStag, a phylogenetic tree was constructed using the amino acid sequence of DjStag and other representative members of the STAG/STAG-like proteins by a neighbor-joining method, along with Homo sapiens STAG as the outgroup (Figure 2). The tree indicated that DjStag is intermediate between insect STAG and amphibian STAG, forming a group with schistosome STAG.

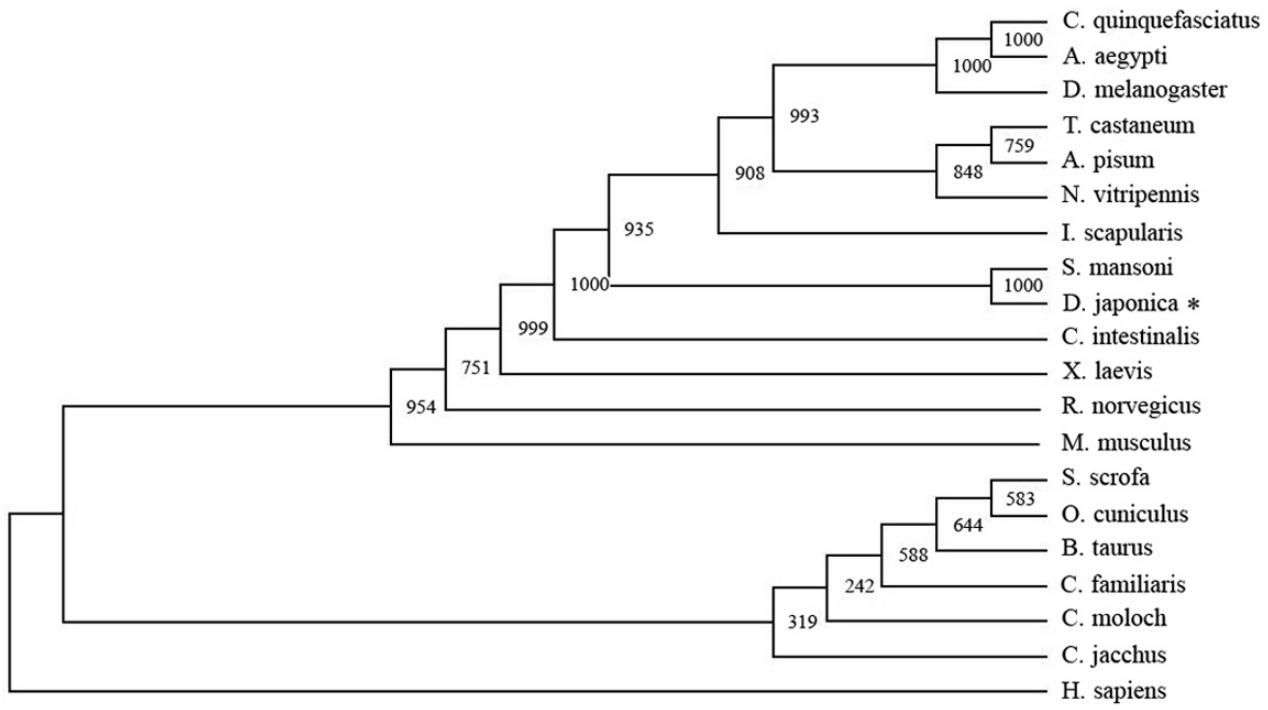

Figure 2. Phylogenic tree of STAG proteins constructed by the neighbor-joining method within the package PHYLIP $3.5 \mathrm{c}$ software package. Homo sapiens STAG was used as outgroup. The numbers refer to bootstrap values of 1000 replicates. GenBank accession numbers of the sequences used are as follows: Homo sapiens, NP_001036214; Callithrix jacchus, ABY90126; Callicebus moloch, ACA57925; Canis familiaris, XP 549232; Bos taurus, XP_001249706; Oryctolagus cuniculus, ACJ76606; Sus scrofa, XP_001926026; Mus musculus, CAM20006; Rattus norvegicus, XP_233108; Xenopus laevis, NP 001080997; Ciona intestinalis, XP_002121258; Schistosoma mansoni, CAZ30922; Ixodes scapularis, EEC02719; Nasonia vitripennis, XP_001600957; Acyrthosiphon pisum, XP_001950748; Tribolium castaneum, XP_966898; Drosophila melanogaster, CAA74654; Aedes aegypti, XP_001648855; Culex quinquefasciatus, XP_001851442.

\section{DjStag is expressed in D. japonica developing embryos}

Northern blotting was conducted to assess the presence and size of the DjStag transcript. As shown in Figure 3, a transcript of approximately 1400 bp was detected, which proved that the gene we obtained was in the planarian D. japonica.

Freshwater planarians (Platyhelminthes: Tricladida) are divided into three families: Dugesiidae, Planariidae and Dendrocoelidae. Studies on the embryonic development of the family Dugesiidae were mainly pursued with Schmidtea polychroa (Bennazzi and Gremigni, 1982; Cardona et al., 2005, 2006). D. japonica belongs to the family Dugesiidae, whose members lay ectolecithal capsules. Several fertilized zygotes are deposited in egg capsules surrounded by large numbers of yolk cells. Cardona et al. (2005) defined an eight-stage system of embryonic development of triclads based on morphology. The embryonic development of $D$. japonica was similar with other triclad species. Thus, the eight stages were adopted in this paper. 


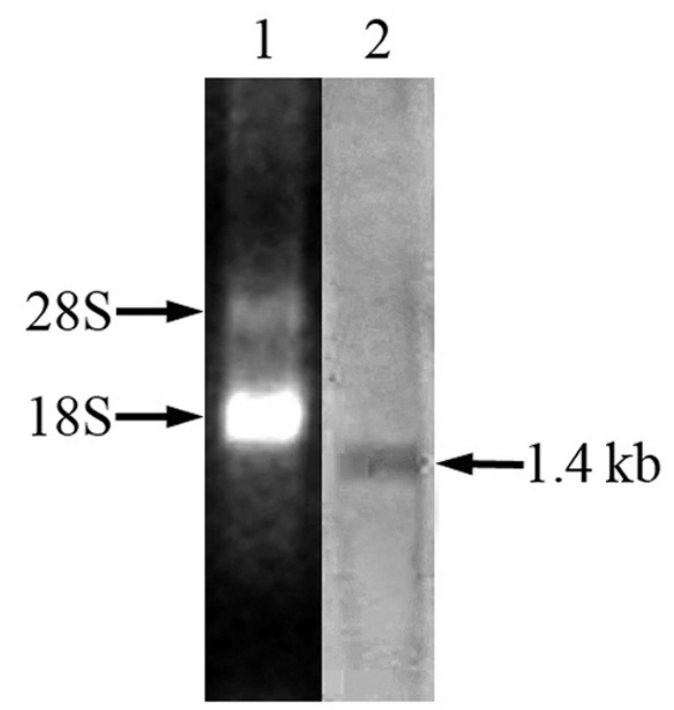

Figure 3. Northern blotting to detect DjStag RNA. Lane $1=$ total RNA of planarians. 28S and 18S rRNA bands are indicated. Lane 2 = the blot was hybridized with DIG-labeled DjStag RNA probe. The arrow indicates the position of molecular size equivalent to $1.4 \mathrm{~kb}$.

DjStag expression pattern in D. japonica during embryonic development was monitored by whole-mount in situ hybridization (Figure 4). The expression of DjStag mRNA was detected in the periphery of stage 3 embryos and in the embryonic pharynx (Figure 4a,b). At this stage, the primary epidermis has formed, covering the whole syncytium. The newly formed embryonic pharynx starts to ingest the external yolk cells into the syncytium. Due to this ingestion, a yolk-filled cavity is formed in the inner part of the embryo and the syncytium is restricted to the periphery of the embryo, which is called the germ band (Cardona et al., 2005; Solana et al., 2009).

During stage 4, embryos grow in size by continued yolk incorporation into the gut cavity (Cardona et al., 2005). DjStag-positive embryonic cells were found in the germ band (Figure 4c,d). In stage 5, embryonic cells start to differentiate into all definitive cell types. Due to the differentiation of muscle cells, previously spherical embryos flatten to a fat disk (Cardona et al., 2005; Solana and Romero, 2009). The number of DjStag-positive embryonic cells in the peripheral germ band increased (Figure 4e).

In stage 6 , the syncytium has been disrupted and completely filled with embryonic differentiating cells. The embryos are more elongated in the antero-posterior (A-P) axis than in earlier stages (Cardona et al., 2005; Solana and Romero, 2009). In this stage, DjStag was mainly found in the dorsolateral region (Figure 4f,g). During stage 7, the embryo stretches, narrows further and becomes worm-shaped. The brain appears as two bilaterally symmetric hemispheres, and two pigmented eye cups form in the dorsal cortex of the brain (Cardona et al., 2005; Solana and Romero, 2009). DjStag-positive signals were detected in the dorsolateral regions along the A-P axis (Figure $4 \mathrm{~h}, \mathrm{i}, \mathrm{j}$ ).

In stage 8 , the embryos show all the juvenile characteristics. The eyes, integument, musculature, nerve plexus, pharynx, and gut take on the characteristics they display in the 
juvenile (Cardona et al., 2005). Expression was observed in the lateral region (Figure 4k), which will eventually become the parenchyma of the adult. At this stage, the embryo moves actively. It will spend a few days in the egg capsule if left undisturbed, but survives normally if dissected out (Cardona et al., 2005).
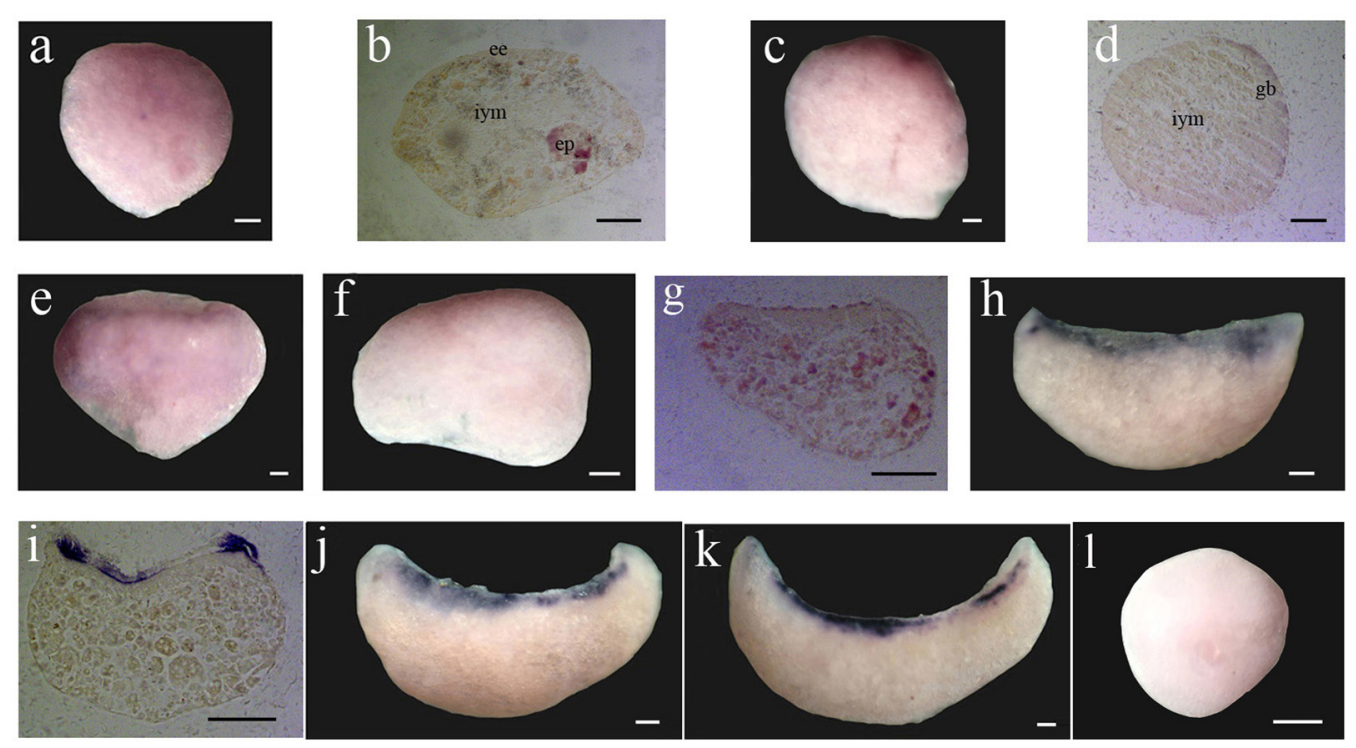

Figure 4. Expression patterns of DjStag mRNA in Dugesia japonica developing embryos detected by whole-mount in situ hybridization. a. Stage 3 embryo and b. section of a stage 3 embryo. The expression of DjStag mRNA is detected in embryonic pharynx and the periphery of embryos. c. Stage 4 embryo and d. section of a stage 4 embryo. DjStag-positive embryonic cells are found in the germ band. e. Stage 5 embryo. The number of DjStag -positive embryonic cells in the peripheral germ band increases. f. Stage 6 embryo and g. section of a stage 6 embryo. The DjStag is mainly found in the dorsolateral region. h. Early stage-7 embryo and i. section of a stage 7 embryo. DjStag-positive signals were detected in dorsolateral regions along the A-P axis. j. Stage 7 later embryo. k. Stage 8 embryo, expression is observed in the lateral region. l. Stage 3 embryo (control) processed and hybridized similarly with sense probes. No signal is seen in the control. In all images, anterior is to the left. ee = embryonic epidermis; $\mathrm{gb}=$ germ band; iym $=$ inner yolk mass; $\mathrm{ep}=$ embryonic pharynx. Scale bars represent $100 \mu \mathrm{M}$.

\section{DjStag is expressed in the juvenile planarians}

Juveniles of $D$. japonica have very similar characteristics as the adult stage. The body wall encloses a thin parenchyma, the brain and nerve cords, the massive pharynx and metameric sets of protonephridia (Cardona et al., 2005). In the juvenile, DjStag mRNA was strongly expressed not only in the head and tail regions, but also in the parenchyma between the epidermis and the gastrodermis (Figure 5a,b,c), which will eventually become the parenchyma of the adult. In addition to this expression along the dorsal side, DjStag was expressed in discrete cells around the branches of the gastrovascular system (Figure 5a).

In triclad adults, much of the space between the gastrodermis and body wall is taken up by the parenchyma. Neoblasts are distributed throughout the planarian parenchyma. In addition, neoblasts are accumulated in multiple clusters along the midline and the lateral lines in 
the dorsal parenchyma (Salvetti et al., 2000; Orii et al., 2005). During regeneration, neoblasts proliferate and accumulate beneath the wound epithelium, giving rise to the regenerative blastema (Salò and Baguñà, 2002; Rossi, et al., 2006). In addition to their role in regeneration, neoblasts are required for homeostasis in intact animals, where they serve to replace damaged or nonfunctional cells (Newmark and Sánchez Alvarado, 2002; Palakodeti et al., 2008).
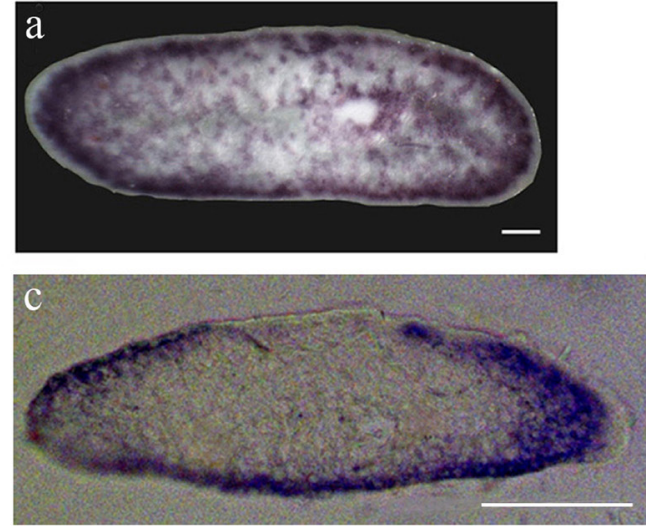
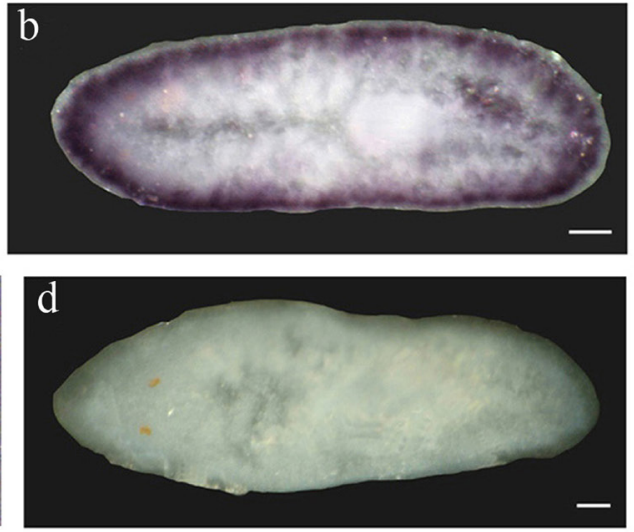

Figure 5. Expression patterns of DjStag mRNA in Dugesia japonica juveniles detected by whole-mount in situ hybridization. a. Juvenile in dorsal view and b. in ventral view; the DjStag mRNA was strongly expressed not only in the head and tail regions, but also in the parenchyma between the epidermis and the gastrodermis. c. Transversal section of a juvenile; the DjStag signals were expressed in the parenchyma. d. Dorsal view of a juvenile planarian (control) processed and hybridized similarly with sense probes. No signal is seen in the control juvenile. In all images, anterior is to the left. Scale bars represent $100 \mu \mathrm{M}$.

Previous study on STAG has been mainly focused on chromatid cohesion and segregation (Lara-Pezzi et al., 2004). In this study, DjStag was shown to be expressed from embryo to juvenile. DjStag was detected in the embryonic cells in the germ band at an early embryo stage. Later, it was mainly expressed in the parenchyma of the lateral region. In the juvenile, the most extensive expression of DjStag was observed in the discrete cells and the parenchyma between the epidermis and the gastrodermis. These results suggest that DjStag is expressed in the cellular subset that will become the neoblast cells of the adult worm. DjStag may play an essential role in cell division during planarian embryonic development.

\section{ACKNOWLEDGMENTS}

Research supported by the National Nature Science Foundation of China (\#31100377, \#41201518), the Shandong Province Natural Science Foundation, China (\#ZR2011CQ018), the Shandong Province Higher Educational Science and Technology Program, China (\#J11LC09) and the Young Teacher Development Support Program of Shandong University of Technology, China. We thank members of our laboratory for suggestions and encouragement.

\section{REFERENCES}

Agata K, Saito Y and Nakajima E (2007). Unifying principles of regeneration I: Epimorphosis versus morphallaxis. Dev. Growth Differ. 49: 73-78. 
Bennazzi M and Gremigni V (1982). Developmental Biology of Triclad Turbellarians (Planaria). In: Developmental Biology of Freshwater Invertebrates (Harrison FW and Cowden RR, eds.). Wiley-Liss, Inc., New York, 151-211.

Cardona A, Hartenstein V and Romero R (2005). The embryonic development of the triclad Schmidtea polychroa. Dev. Genes Evol. 215: 109-131.

Cardona A, Hartenstein V and Romero R (2006). Early embryogenesis of planaria: a cryptic larva feeding on maternal resources. Dev. Genes Evol. 216: 667-681.

Hirano T (2002). The ABCs of SMC proteins: two-armed ATPases for chromosome condensation, cohesion, and repair. Genes Dev. 16: 399-414.

Kozak M (1987). An analysis of 5'-noncoding sequences from 699 vertebrate messenger RNAs. Nucleic Acids Res. 15: $8125-8148$.

Lara-Pezzi E, Pezzi N, Prieto I, Barthelemy I, et al. (2004). Evidence of a transcriptional co-activator function of cohesin STAG/SA/Scc3. J. Biol. Chem. 279: 6553-6559.

Lee JY and Orr-Weaver TL (2001). The molecular basis of sister-chromatid cohesion. Annu. Rev. Cell Dev. Biol. 17: 753-777.

Liu Z, Zhang S, Yuan J, Sawant MS, et al. (2002). Molecular cloning and phylogenetic analysis of AmphiUbf80, a new member of ubiquitin family from the amphioxus Branchiostoma belcheri tsingtauense. Curr. Sci. 83: 50-53.

Losada A, Hirano M and Hirano T (1998). Identification of Xenopus SMC protein complexes required for sister chromatid cohesion. Genes Dev. 12: 1986-1997.

Losada A, Yokochi T, Kobayashi R and Hirano T (2000). Identification and characterization of SA/Scc3p subunits in the Xenopus and human cohesin complexes. J. Cell Biol. 150: 405-416.

Michaelis C, Ciosk R and Nasmyth K (1997). Cohesins: chromosomal proteins that prevent premature separation of sister chromatids. Cell 91: 35-45.

Nasmyth K (2001). Disseminating the genome: joining, resolving, and separating sister chromatids during mitosis and meiosis. Annu. Rev. Genet. 35: 673-745.

Nasmyth K, Peters JM and Uhlmann F (2000). Splitting the chromosome: cutting the ties that bind sister chromatids. Science 288: 1379-1385.

Newmark PA and Sánchez Alvarado A (2002). Not your father's planarian: a classic model enters the era of functional genomics. Nat. Rev. Genet. 3: 210-219.

Orii H, Sakurai T and Watanabe K (2005). Distribution of the stem cells (neoblasts) in the planarian Dugesia japonica. Dev. Genes Evol. 215: 143-157.

Palakodeti D, Smielewska M and Graveley BR (2006). MicroRNAs from the Planarian Schmidtea mediterranea: a model system for stem cell biology. RNA 12: 1640-1649.

Palakodeti D, Smielewska M, Lu YC, Yeo GW, et al. (2008). The PIWI proteins SMEDWI-2 and SMEDWI-3 are required for stem cell function and piRNA expression in planarians. RNA 14: 1174-1186.

Prieto I, Suja JA, Pezzi N, Kremer L, et al. (2001). Mammalian STAG3 is a cohesin specific to sister chromatid arms in meiosis I. Nat. Cell Biol. 3: 761-766.

Reddien PW and Sánchez Alvarado A (2004). Fundamentals of planarian regeneration. Annu. Rev. Cell Dev. Biol. 20: 725-757.

Reddien PW, Bermange AL, Kicza AM and Sánchez Alvarado A (2007). BMP signaling regulates the dorsal planarian midline and is needed for asymmetric regeneration. Development 134: 4043-4051.

Rossi L, Salvetti A, Lena A, Batistoni R, et al. (2006). DjPiwi-1, a member of the PAZ-Piwi gene family, defines a subpopulation of planarian stem cells. Dev. Genes Evol. 216: 335-346.

Rubio ED, Reiss DJ, Welcsh PL, Disteche CM, et al. (2008). CTCF physically links cohesin to chromatin. Proc. Natl. Acad. Sci. U. S. A. 105: 8309-8314.

Salò E and Baguñà J (2002). Regeneration in planarians and other worms: New findings, new tools, and new perspectives. J. Exp. Zool. 292: 528-539.

Salvetti A, Rossi L, Deri P and Batistoni R (2000). An MCM2-related gene is expressed in proliferating cells of intact and regenerating planarians. Dev. Dyn. 218: 603-614.

Solana J and Romero R (2009). SpolvlgA is a DDX3/PL10-related DEAD-box RNA helicase expressed in blastomeres and embryonic cells in planarian embryonic development. Int. J. Biol. Sci. 5: 64-73.

Solana J, Lasko P and Romero R (2009). Spoltud-1 is a chromatoid body component required for planarian long-term stem cell self-renewal. Dev. Biol. 328: 410-421.

Ström L, Lindroos HB, Shirahige K and Sjogren C (2004). Postreplicative recruitment of cohesin to double-strand breaks is required for DNA repair. Mol. Cell 16: 1003-1015.

Ström L, Karlsson C, Lindroos HB, Wedahl S, et al. (2007). Postreplicative formation of cohesion is required for repair and induced by a single DNA break. Science 317: 242-245. 
Sumara I, Vorlaufer E, Gieffers C, Peters BH, et al. (2000). Characterization of vertebrate cohesin complexes and their regulation in prophase. J. Cell Biol. 151: 749-762.

Wang Y, Zhao B, Zhang S and Qu X (2008). Characterization, expression and localization of S-adenosylhomocysteine hydrolase from amphioxus Branchiostoma belcheri tsingtaunese. Biosci. Rep. 28: 135-144. 